\title{
Real Time Implementation for Monitoring Drowsiness Condition of a Train Driver using Brain Wave Sensor
}

\author{
Upasana Sinha \\ Dr.C.V.Raman University \\ Department of Computer \\ Science Engineering \\ Bilaspur, India
}

\author{
Kamal K. Mehta, PhD \\ Nirma Institute of Technology \\ Department of Computer \\ Science Engineering \\ Ahemedabad, India
}

\author{
A.K. Shrivastava, PhD \\ Dr.C.V.Raman University \\ Department of Physics \\ Bilaspur, India
}

\begin{abstract}
Driver fatigue and lack of sleep of drivers especially those who drive for a longer period of time as train accidents are a longer standing problem. It has been observed that each year numerous train accidents and fatalities may occur around the world due to driver falling asleep while driving the train. There are various traditional methods that may facilitate to detect drowsiness state of the driver to warn in such a manner, so that such accidents may be prevented to large extent. In this implementation, a system for determining drowsiness state of a driver to avoid an accident is disclosed. In one aspect, the system comprises a brainwave sensor, a microcontroller, and an alarm unit. The sensor which is the Brain Computer Interface (BCI) may be attached to one or more touch points for sensing a brainwave emitted by neurons in a brain of the driver. The microcontroller, coupled with the sensor, configured to analyze the mind in order to determine a category of the brainwaves. The brainwave may be categorized into the category based upon a predefined frequency range associated with the brainwave of types Delta, Theta, Alpha and Beta. The in-built Bluetooth will be paired up with the microcontroller to start messaging regarding the status of the driver through the GSM modem, and soon after it will alarm in the motor or in nearby station if required.
\end{abstract}

\section{General Terms}

The general classification of the submitted material is based on Artificial Intelligence, Security.

\section{Keywords}

Brain wave sensor (BCI), Railway derailment accident, EEG signal processing.s

\section{INTRODUCTION}

Drowsy and fatigue driving is a major transportation safety concern and is responsible for thousands of accidents and numerous fatalities every year. Drowsy driving crashes are usually of high severity due to the driver's significant loss of control, often leading to unpredicted vehicle trajectory and no braking response. Reliable safety systems are needed to mitigate these crashes. The most important challenge is to detect the driver's condition sufficiently early, prior to the onset of sleep, to avoid collisions. An electroencephalogram (EEG) is a test during which the electric signals of the brain are measured from the scalp of the user. An EEG based brain computer interface (BCI) has been used for various applications including the psychological status of the driver. The reason to choose Railways is quite unique and distinctive in character, really a microcosm of India. Railways are large infrastructure and are the prime modes of transportation in many countries. There is always some risk associated with derailments but it can be reduced by elimination of the root causes. A derailment is an accident on a Railway in which a train leaves the rails, which can result in damage, injury and death. In this paper mind wave sensor is used which is the Brain Computer Interface (BCI) device look like the headset which turns the brain waves into actions, unlocking new worlds of interactivity. This process is unique as the processing time is very less rather than in using laptops or cameras. This technique is applied for detecting drowsiness is by monitoring the response of the driver using head sensor. The working of this method includes the sensor that touches the forehead, the contact and reference points located in the ear clip, and the on-board chip that process all of the data. The two standard modes i.e. Active mode and Rest mode will be analyzed by the voltage references and will start comparing with the database which will be stored in the AVR (Advanced Virtual RISC) Microcontroller. The in-built Bluetooth will be paired up with the AVR microcontroller to start messaging with it about the Active and Rest mode. As soon as it gets the message about the modes, it will buzz the alarm in particular location of the driver's cabin.

\section{RELATED WORK}

Railway transport is one of the major modes of transportation, so it must offer high comfort level for the passengers and the staff. In depth study of human fatigue has only occurred during the last twenty five years. The Scientific community has discovered the structure of sleep, the role circadian rhythms and the effects of sleep deprivation. Researchers have also investigated the benefits of sleep management including a nappy study by Dinges (1995). According to National Sleep Foundation's 2005 Sleep in America poll [10,2], 60\% of adults drivers about 168 million people say they have driven a vehicle while feeling drowsy in the past year, and more than one third, (37\% or 103 million people), have actually fallen asleep at the wheel. Earlier studies of Rail transportation have reported an increase of sleepiness during the span of a night shift (a combined effect of the night shift and shift length). During both simulated and true train driving, physiological sleepiness (alpha and theta activity of the EEG) increases steadily during a night shift (Fruhstoffer et al. 1977, Torsvall and Akerstedt 1987). Similarly increase in physiological sleepiness and intrusions of normal sleep into periods of wakefulness were most frequent during the second half of a night shift in industrial process operators (Torsvall et al. 1989) $[2,3]$.Many methods have been developed to detect fatigue in train drivers ever since they began and methods were introduced to try to prevent them. With great development in the Railway system and the continuous input of new technologies to the Railway transportation safety, the work timings of the pilots are not flexible. Due to all these factors, fatigue driving is becoming a big issue. Some of the related works in this area are mentioned below: 


\subsection{PIR (Passive Infrared Sensor) and MEMS (Micro-electromechanical Sensor)}

It is described that the sensors detect whether the pilot is awake, sleepy and drowsy and controls the speed of train for safety purpose. The aim of the research is that the system is to measure precisely the development and progression of driver fatigue and loss of alertness, and to provide counter measure to address the danger [5]. Thus the guarantee and supervision of the system has to be ensured and so the status is also transferred to the nearby location. A LCD (liquid crystal display) is an image displaying technology with 16x2 alphanumeric dot matrix displays is used to show the status.

\subsection{Face Detection flow based on Haar Classifiers}

In this research it is shown that first driver drowsiness of all detection has focused on medical science, which is done on the basis of medical electroencephalograph (EEG), electrocardiograph (ECG) and electromyography (EMG) to detect a driver's EEG waveform. The method is based on Image processing [7]. In reference, Qiang Ji et al used Kalman filtering for eye tracking method; it is a linear system estimation algorithm. In fatigue detection eye motion has the highly nonlinear so standard Kalman filter is no longer favorable.

\subsection{Detection of Drowsiness using \\ Representation Learning}

Here, it is described that autonomous systems designed to analyze driver exhaustion and detecting driver drowsiness can be an integral part of the future intelligent vehicle to prevent accidents caused by sleep. In this Research it has been suggested that as the alertness level decrease EEG power of the alpha and theta bands increases [11], hence providing facts of drowsiness. New techniques are based on machine learning algorithms to detect driver drowsiness levels. Vural et al. [11, 12] creates Automatic classifier for 30 facial actions from the Facial Action Coding system using machine learning on a separate database of spontaneous expressions to finally categorize driver drowsiness. Here, frames are extracted from the video. These frames are fed to a Viola and Haar like features based face detector. The detected faces are cropped and images are normalized by subtracting each pixel by the mean followed by division with its standard deviation. Normalized images of $80 \%$ subjects are further fed to multilayer convolutional neural networks. The output of the hidden layer is considered as the extract features. On the basis of these features, the softmax layer classifier was trained. Once the classifier has been trained, the rest $20 \%$ of the images extracted earlier are tested on the trained classifier.

\subsection{Some of the Related Patents and its Applications have also been studied regarding this problem.}

There are many patents which are studied regarding this work but three patents are considered as base paper like CN102073857 published in year 2011 [17] are referred. In this research the concrete steps of the method falls in two categories, first it acquires dual image and secondly extracting image sequence of face parts and applied template matching. The other US7830266 publication in the year 2009 [18] stated that a blink detection section generates an eye closure signal indicating the duration of a period for which the driver closes his/her eyes. A drowsy state determination section compares the respective occurrence frequencies with the first eye closure frequency threshold and the second eye closure frequency threshold to determine which of the drowsiness levels corresponding to the respective thresholds the drowsiness level of the driver is at. The third patent US6717518 publication in the year 2004 [19] shows that the process of detecting a person fallen asleep, an image of the face is acquired. Pixels of the image having characteristics corresponding to an eye of the person are selected and a histogram is formed of the selected pixels. The histogram is analyzed over time to identify each opening of the eye, and characteristics indicative of the person falling asleep are determined.

\section{METHODOLOGY ADOPTED}

Before discussing about the research method, it is well understood that the entire previous base papers are based on Image processing and Image acquisition. In this research it is overcome by focusing on the Mind waves and everything is directly connected to it. Brain Wave sensor which is the Brain Computer Interface (BCI) is a device which communicates using thoughts of Brain (EEG) without using any Muscle control.

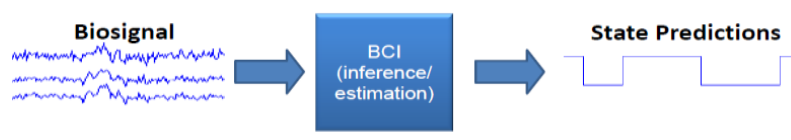

Fig 1: Brain computer interface

The last century of the research has greatly increased our knowledge about the brain and particularly the electrical signals emitted by neurons firing in the brain. The patterns and frequencies of these electrical signals can be measured by placing a sensor on the scalp. To detect high drowsiness effect, one can depend on the following types:

Table 1: Brain waves classification

\begin{tabular}{|c|c|c|c|}
\hline S.No. & $\begin{array}{c}\text { Brainwave } \\
\text { Type }\end{array}$ & $\begin{array}{c}\text { Frequency } \\
\text { range }\end{array}$ & $\begin{array}{c}\text { Mental } \\
\text { states and } \\
\text { conditions }\end{array}$ \\
\hline 1 & Delta & $\begin{array}{c}0.1 \mathrm{~Hz} \text { to 3 } \\
\mathrm{Hz}\end{array}$ & $\begin{array}{c}\text { Deep, } \\
\text { dreamless } \\
\text { sleep, } \\
\text { unconscious }\end{array}$ \\
\hline 2. & Theta & $\begin{array}{c}4 \mathrm{~Hz} \text { to } 7 \\
\mathrm{~Hz}\end{array}$ & $\begin{array}{c}\text { Intuitive, } \\
\text { creative, } \\
\text { recall, } \\
\text { fantasy, } \\
\text { imaginary, } \\
\text { dream }\end{array}$ \\
\hline 3. & Alpha & $8 \mathrm{~Hz}$ to 12 \\
$\mathrm{~Hz}$ & $\begin{array}{c}\text { Relaxed(but } \\
\text { not drowsy) }\end{array}$ \\
\hline 4. & Beta & $\begin{array}{c}21 \mathrm{~Hz} \text { to } \\
30 \mathrm{~Hz}\end{array}$ & $\begin{array}{c}\text { Alertness, } \\
\text { agitation. }\end{array}$ \\
\hline
\end{tabular}




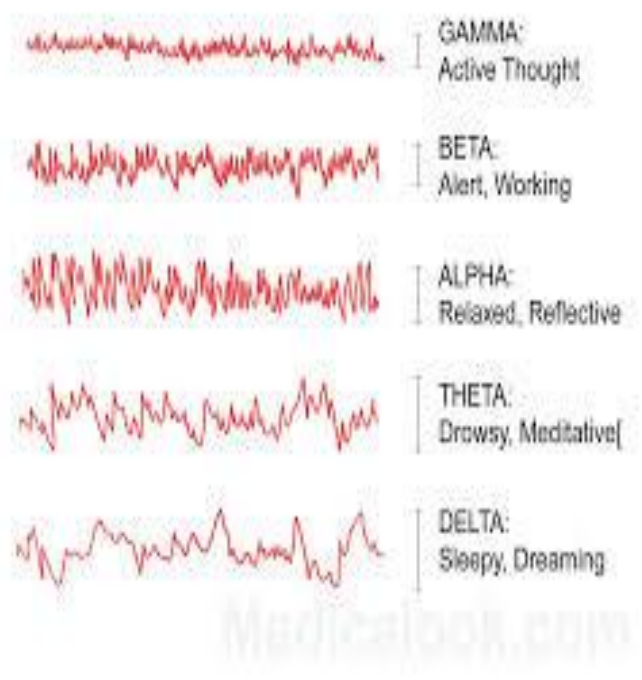

The category of the brain waves and its signals that is mentioned above is defined by EEG (Electroencephalography). The drowsiness condition comes under the term Micro sleep. It is the temporary episode of sleep which may last for a fraction of second or up to 30 seconds, and it leads to fail individuals to respond at certain point, and this particular point is measured by the brain wave sensor after having simulation with the hardware. The brain wave sensor has to wear in such a manner that touches the forehead, the contact and reference points located in the ear clip, and the on-board chip (Bluetooth) that processes all the data. The goal is to build a brain computer interfacing using an AVR Microcontroller. The purpose is to build a low cost alternative that would allow the users to take their health in their own hands by diagnosing and attempting to treat their own sleep disorders and the Mind wave sensor an EEG gadget that safely measures and transfer power spectrum (alpha waves, beta waves etc.). This can be simply slipped on to be able to see levels of attention and relaxation and even learn about how the brain responds. This can be an excellent introduction to the world of brain-computer interface. As it is already mentioned that this research is not using any PC or laptops as to increase process time the AVR Microcontroller will be used as this is much simpler and deals with data in 8 bit chunks. A program is a series of instructions, each very simple, that fetches and manipulates data. In most applications, where AVR is used, it means reading inputs, checking their state and switching on outputs accordingly.

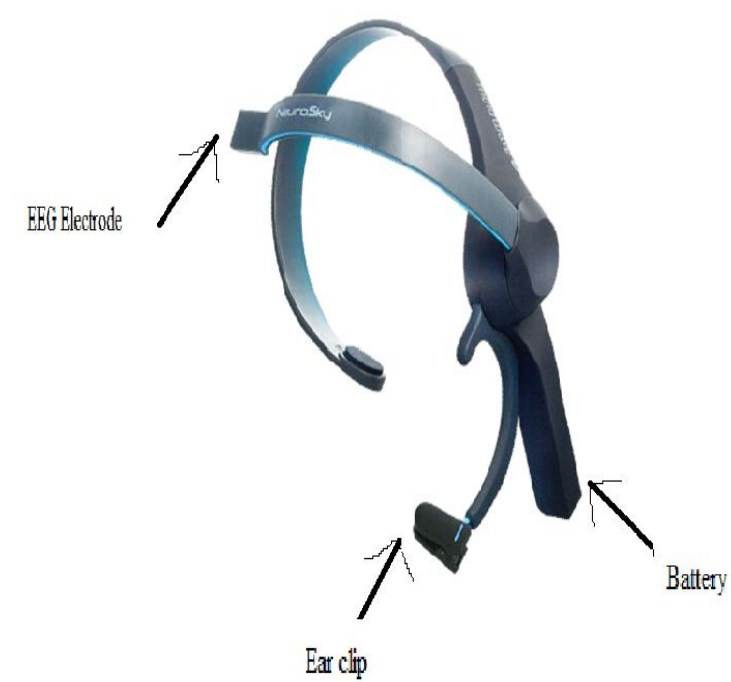

Fig 2: Mind wave sensor

The sensor is single channel, wireless headset that monitors the brain activity and translates EEG into meaning data that anyone can understand. The specifications of the sensor are as follow:

i. Bluetooth ${ }^{\mathrm{TM}}$ Wireless communications

ii. Passive Dry Sensor EEG

iii. Triple AAA battery

eSense Brainwave Patterns:

i. If the person is in Attention mode.

ii. It can measure the meditation level and provides reports to analyze.

iii. The Eye Blinks detection, real time Brain monitoring by sensing its alertness.

iv. Its Frequency Bands ranges from $0.5-50 \mathrm{~Hz}$.

\subsection{Work Flow Diagram}

Here it may be seen that there is an interfacing between the Bluetooth of mind wave sensor and the hardware. The transmission speed of Bluetooth is 9600 bps (bits per second) and the transmission speed of Bluetooth of Mind wave is $57600 \mathrm{bps}$, so for this the calibration is required to make transmission speed high of hardware from $9600 \mathrm{bps}$ to 57600bps. This calibration is known as Command mode 
Driver side

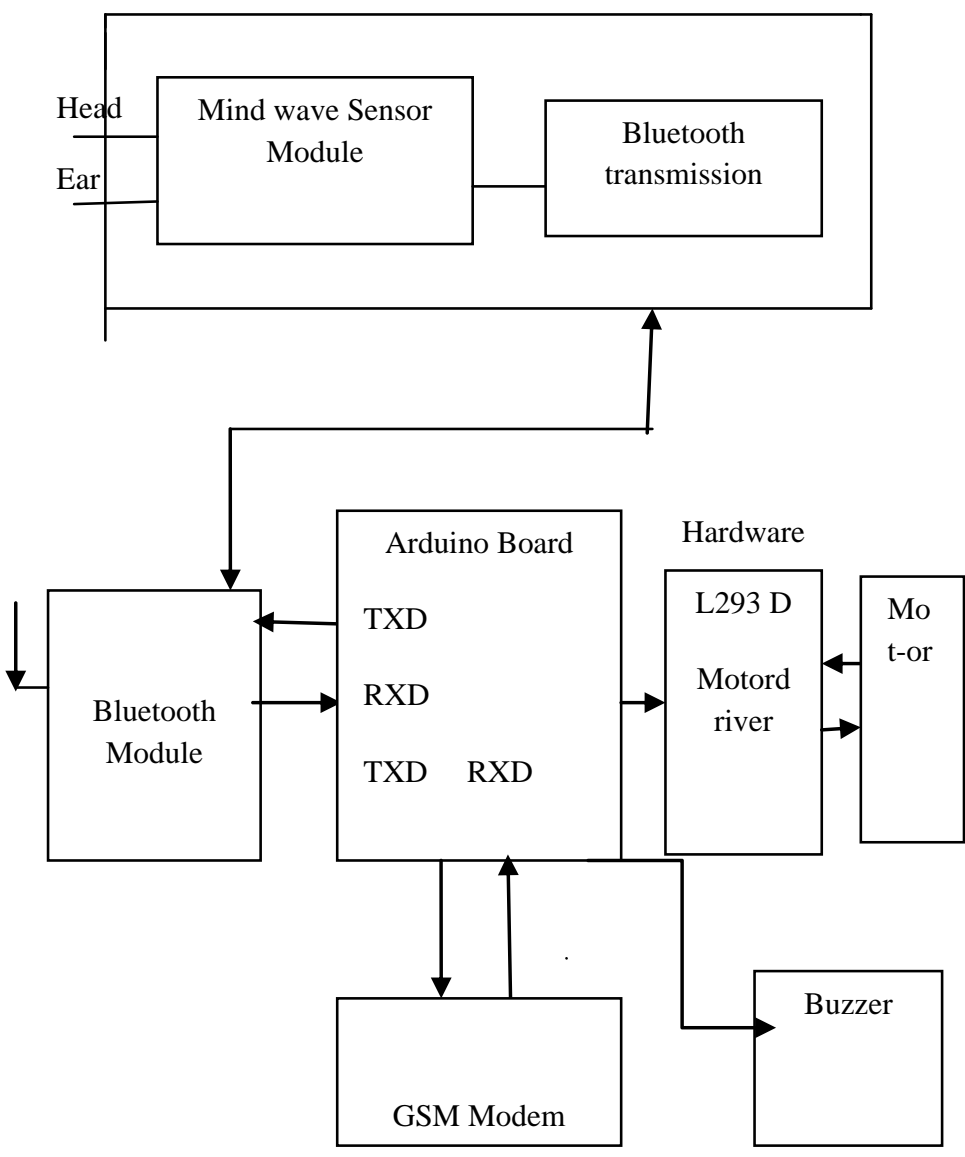

Fig 3: Architecture of the system.

AT Commands for HC-05 Module Initialize for Mind wave Sensor

Initially, the sensor has to be calibrated with the Bluetooth using command mode and then the following commands for HC-05 module to communicate the mind wave sensor.

\author{
$\mathrm{AT}+\mathrm{Name}=$ "Upasana" \\ AT+UART="57600,0,0" \\ AT + Role $=" 1 "($ Text mode $)$ \\ $\mathrm{AT}+\mathrm{PSWD}=" 0000 "$ \\ $\mathrm{AT}+\mathrm{CMODE}=" 0 "$ \\ $\mathrm{AT}+\mathrm{BIND}=" 2068$,9D, 4c1472" (Mind wave unique number) \\ $\mathrm{AT}+\mathrm{IAC}=" 9 \mathrm{E} 8 \mathrm{~B} 33 "$ \\ $\mathrm{AT}+\mathrm{CLASS}=" 0 "$ \\ $\mathrm{AT}+\mathrm{INQM}=" 1,9,48 "$
}

After making this, the Bluetooth will be paired up with each other and sensor will start working. Here, the Bluetooth hardware is connected with the Arduino board and the one small motor driver is attached to it. Arduino is an open source electronics platform based on easy to use hardware and software. It's intended for anyone making interactive projects. Arduino senses the environment by receiving inputs from many sensors, and affects its surroundings by controlling light, motors and other actuators. There is a GSM modem is used to convey the message regarding "chance of accident" if the driver feels drowsiness and immediately the buzzer will sound. The frequency of the sensor has been set below $30 \mathrm{~Hz}$ in danger zone, and sound will buzz 5 times to alert the driver.

The LED (light emitting diode) is used in the board to indicate the condition of the driver, if it is ON then the quality is poor and if it is OFF, the status is good. It is basically used to monitor the attention and poor quality of the driver.

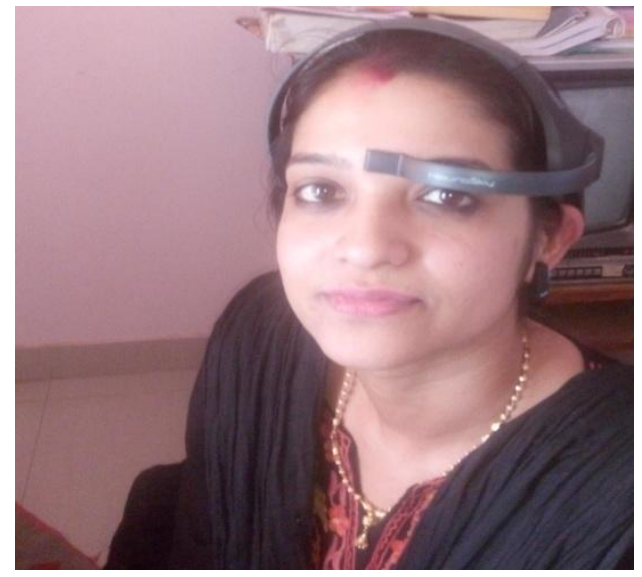

Fig 4: Mind wave sensor attached from 3 points.

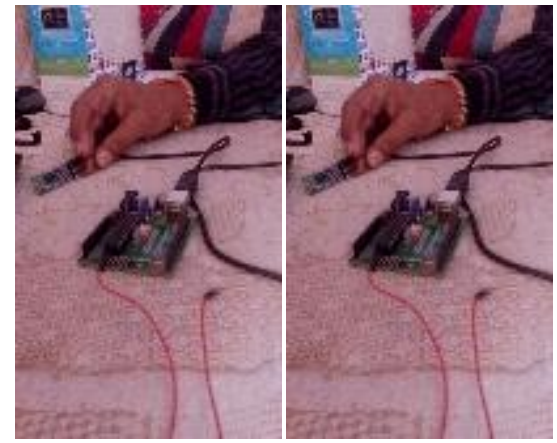

Fig 5: Bluetooth device interfacing with the Microcontroller.

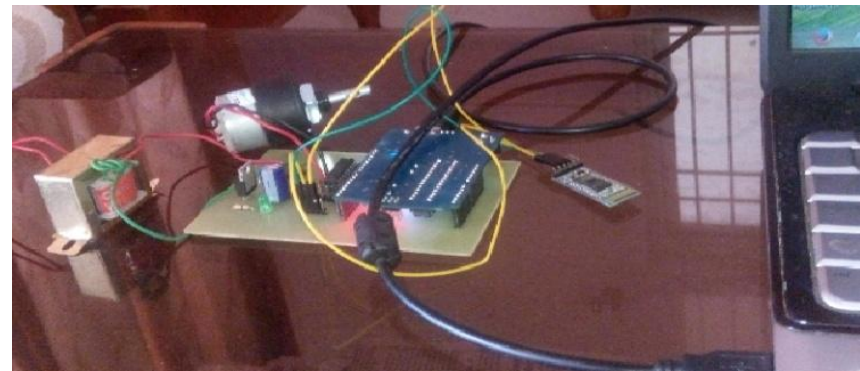

Fig 6: Hardware interfacing with the system 


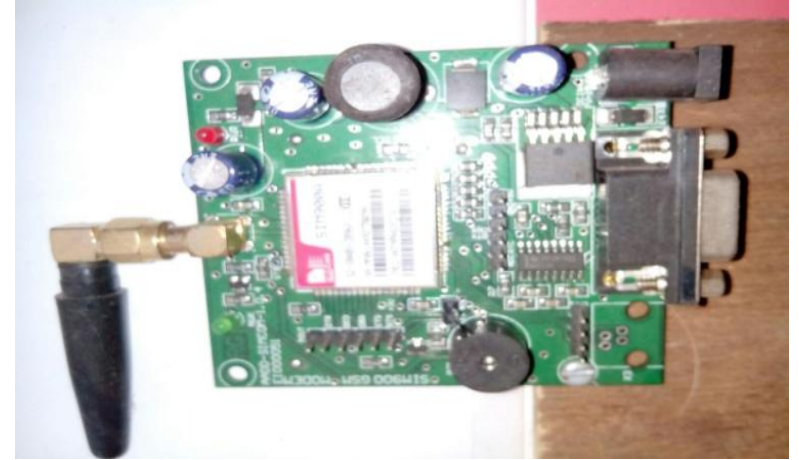

Fig 7: GSM Modem

A GSM (Global system for mobile communication) is also used which is a specialized type of modem which accepts a SIM card and operates over a subscription to a mobile operator, just like a mobile phone. When a GSM modem is connected to hardware, this allows the computer to use the GSM modem to communicate over the mobile network. It will help to prevent accident by delivering message to the nearby station.

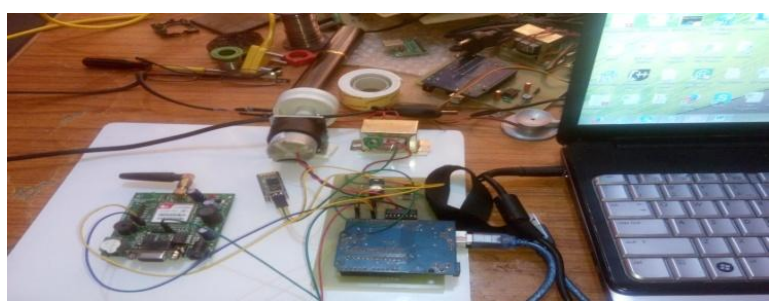

Fig 8: Hardware implementation with the PC.

\section{RESULT}

The output describes about the frequencies of the brain. If the poor quality of the brain shows zero that means attention is high and vice versa. But if the attention is low, poor quality is high.

The poor quality is one of the variable that is been assigned to track the quality of the signal, if it is low that means the range of the signal is able to track the condition of the driver in a proper manner. Similarly, as soon as it gets high the attention mode becomes zero, and then the alarming condition arises.

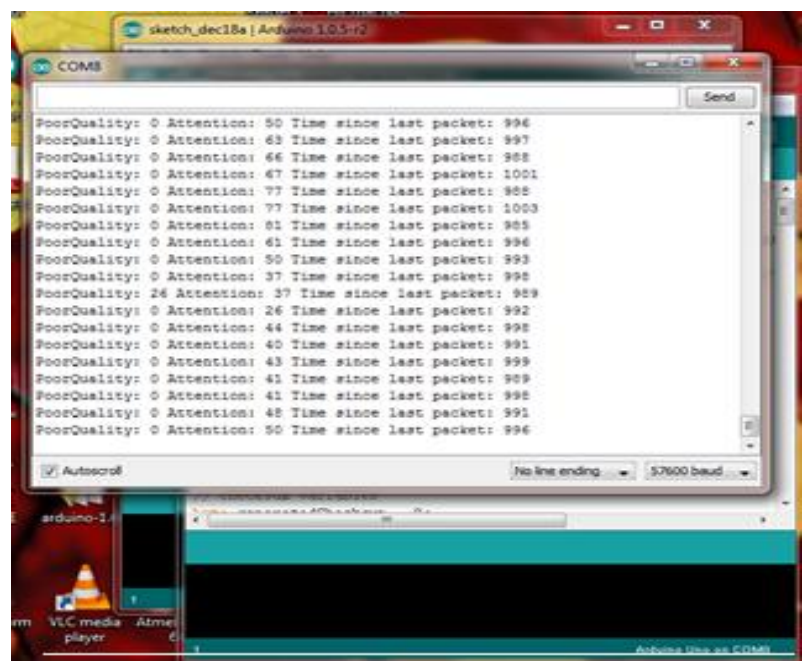

Fig 9: The output the brain sensor with respect to poor quality and attention mode in awake condition

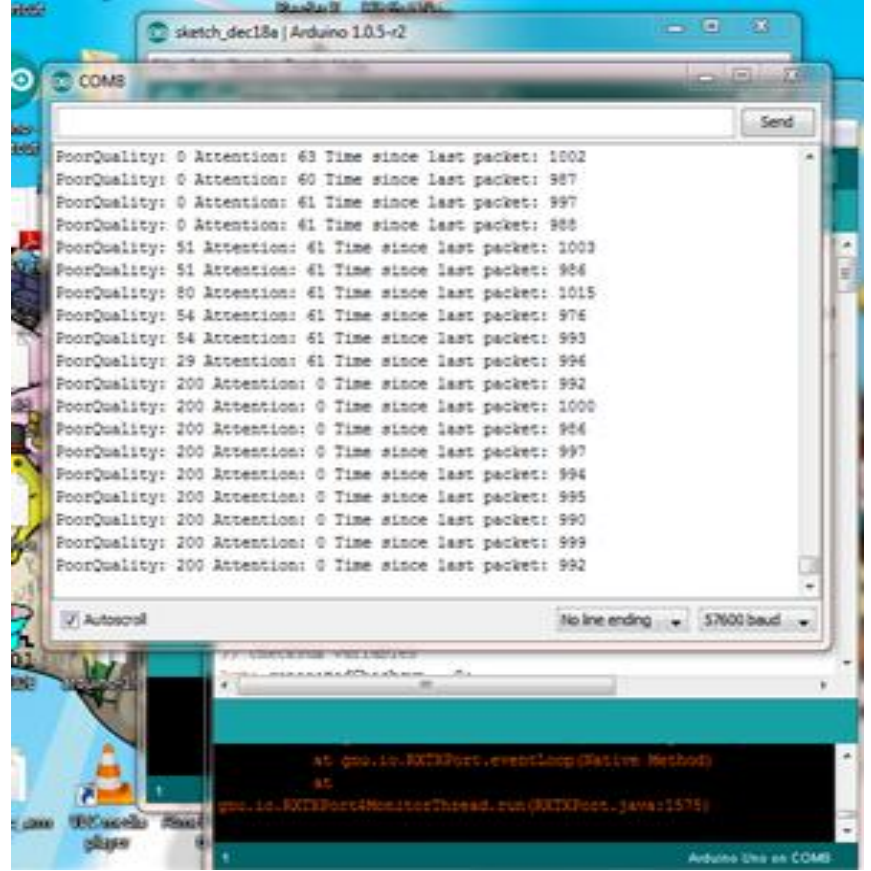

Fig 10: The output of the brain sensor in drowsiness condition

In the above result it is clearly shown that when the poor quality is low attention is high and when the poor quality is high the attention is low. The readings are clearly available for every second of state of mind and it is already mentioned that if the frequency the attention position goes below $30 \mathrm{~Hz}$, it indicates danger zone which will be real time status of the drive. The actual status will come out as it directly scans the brain system.

\section{FEATURES}

Single sensor on FP1 (Forehead frontal point), as this is a forehead area with minimal hair, it offers EEG clarity to enable the accurate delivery of result. Different brain states are the result of different patterns of neural interaction. As example, brainwaves between $12 \mathrm{~Hz}$ to $30 \mathrm{~Hz}$ are called Beta waves, they are associated with concentration, while waves between $8-12 \mathrm{~Hz}$ are called Alpha waves, and they are associated with calm relaxation. The contraction of muscle is also associated with unique wave patterns, called EMG waves. Isolating EMSG patterns Mind wave device detect eye blink. The Reference electrode on Ear clip to remove ambient noises. The earlobe is a location that experiences the same ambient noise as the Neurosky forehead sensor but with minimal neural activity. IP involves cancellation and signal amplification. Extrapolating EEG brainwave signals from noise requires both a reference point and electrical circuit grounding. The grounding makes the body voltage the same as the headset. Provides EMG (Electromyography) feature for Eye blink detection.

\section{Advantages}

One will get the actual real time readings as the sensor directly scans the brain. It is real time hardware implementation; status updating is done in a fast manner. It is less delayed as the processing speed is high.

\section{Limitations}

The battery of the sensor has to be changed after every 7-8 hours in a day as to operate the sensor in an appropriate manner. 


\section{Applications}

It is used to detect braking intentions before the driver depresses the brake pedal by EEG and EMG sensors. The Lane-change intentions can also be done as the reliability of steering behavior analysis by detecting driver fatigue. The Workload/fatigue/Alertness monitoring in Drivers, Pilots is also done.

\section{REFERENCES}

[1] Budiharto, W. \& Putra,W. (2013). Design and Analysis of Fast Driver's Fatigue Estimation and Drowsiness Detection System using Android. Journal of Software, vol.8, no.12, pp.3055-3059.

[2] Gulhane, Miss. M., \& Mohod, P.S. (2013). INTELLIGENT FATIGUE DETECTION AND AUTOMATIC VEHICLE CONTROL SYSTEM. International Journal of Computer Science \& Information Technology (IJCSIT), vol. 6, No.3, pp. 87-92.

[3] HARMA, M. \& SALLINEN, M. et al. (2002).The effect of an irregular shift system on sleepiness at work in train drivers and railway traffic controllers. J. Sleep Res., vol.11, pp.141-151.

[4] Lal, S.K., et al. (2003). Development of an algorithm for an EEG- based driver fatigue countermeasures", Journal of safety Research, vol.34, pp. 321-328.

[5] Sende P. \& Warade, et al. (2015). Driver Fatigue Detection System and the Status Transmission. International Journal of Innovative Research in Science, Engineering and Technology, vol.4, no.6, pp. 4923-4927.

[6] Sontakke, K. (2015). Efficient Driver Fatigue Detection and Alerting System. International Journal of Scientific and Research Publications, vol. 5 , no. 7, pp.1-4.

[7] Tayade, M. R. et al. (2014). Real Time Eye State Monitoring System for Driver Drowsiness Detection. International Journal of Emerging Technology and Advanced Engineering, vol. 4, Issue 6, pp. 452-456.

[8] Viola, P. \& Jones, M. (2004). Robust Real Time Face Detection. International Journal of Computer Vision, vol.57, no. 2, pp.137-154.

[9] Angeline, P.J., Saunders, G.M. \& Pollack, J.B. (1994). An evolutionary algorithm that constructs recurrent neural networks. IEEE Transactions on Neural Networks, vol.5, pp.54-65.

[10] Dorrian, J., et al.; (2006),Simulated train driving: Fatigue, self-awareness and cognitive disengagement; Applied Ergonomics, vol. 38, pp.155-166.

[11] Dwivedi, K., Biswaranjan, K. \& Sethi, A. (2014). Drowsy Driver Detection using Representation Learning. Advance Computing Conference (IACC), IEEE Conference, Gurgaon, pp.995-999.

[12] Eakandarian, A. \& Mortazavi, A. (2007). Evaluation of a smart algorithm for commercial vehicle driver drowsiness detection. Proceeding of the IEEE intelligent vehicle symposium, pp.553-559.

[13] Jap, B.T. et al. (2007). Using Special Analysis To Extract Frequency Components From Electroencephalography: Application for Fatigue Countermeasure in train drivers. Proc.-IEEE $2^{\text {nd }}$ International Conference on Wireless Broadband and Ultra Wideband Communications, pp.13.

[14] Rau, P.S. (2005). Drowsy driver's detection and warning system for commercial vehicle drivers: Field proportional test design, analysis, and progress. $19^{\text {th }}$ International Technical Conference on the Enhanced safety of Vehicles, Washington, D.C.

[15] Salakhutdinov, R. \& SMnih, A. et al. (2007). Restricted Boltzmann machines for collaborative filtering. Proceeding- $24^{\text {th }}$ International conference on machine learning, pp.791-798.

[16] Smith, P., Shah, M. \& Lobo, N.V. (2000). Monitoring head/eye motion for driver alertness with one camera", Proceedings- $15^{\text {th }}$ International Conference on Pattern Recognition on Pattern Recognition, vol.4.

[17] U. Shenyang Technology; Multimodal driver fatigue detection method and special equipment thereof, Publication no. CN102073857, Published on May 25, 2011.

[18] A. Nagakoshi Toyota (JP), et al.; Drowsy state determination device and method, Publication no. US 7830266, Published on January 29, 2009.

[19] P. Pirim Paris (FR), et al.; Method and apparatus for detection of drowsiness, Publication no. US6717518, Published on April 6, 2004.

[20] Sinha, U., Mehta, K., A system and method for determining Drowsiness condition of a Train Driver, Application no. 2778/MUM/2015, and Filed on July 22, 2015. 